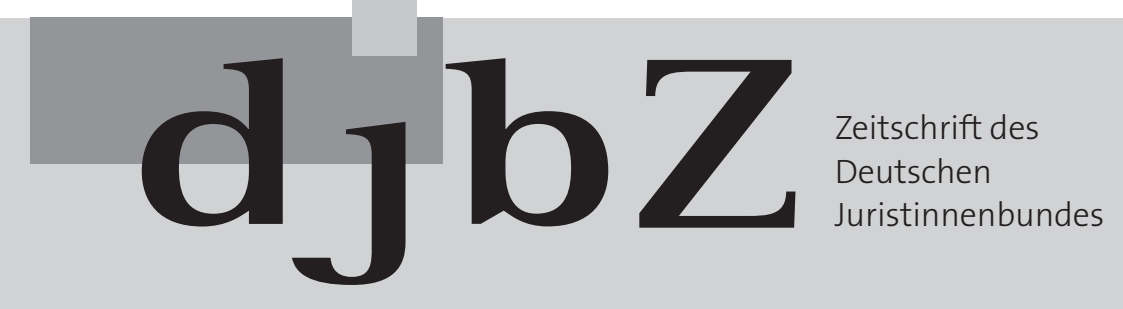

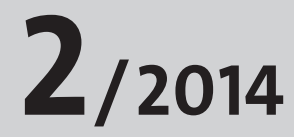

17. Jahrgang, Seite 47-92

Herausgeber: Deutscher Juristinnenbund e. V.

Präsidium: Ramona Pisal, Vorsitzende Richterin am Oberlandesgericht, Brandenburg a.d.H. (Präsidentin); Margarete Hofmann, Direktorin in der EU-Kommission Brüssel; Eva Schübel, Bundesanwältin beim BGH, Karlsruhe (Vizepräsidentinnen);

Dr. Karin E.M. Kopp, LL.M. (Berkeley), Regierungsrätin, Finanzamt München (Schatzmeisterin);

Schriftleitung: Anke Gimbal, Rechtsassessorin, Geschäftsführerin Deutscher Juristinnenbund, Berlin.

\title{
Aktionärinnen fordern Gleichberechtigung 2009 bis 2013 Zum Abschluss des Projektes: „Sag mir, wo die Frauen sind“
}

\author{
Anke Gimbal, Birgit Kersten, Simone Krämer, Katrin Lange, \\ Kivilcim Simsek, Johanna Thumm \\ Projektteam Deutscher Juristinnenbund e.V., Berlin
}

Am 28. November 2013 hat der Deutsche Juristinnenbund e.V. (djb) die abschließenden Ergebnisse seines Projektes „Aktionärinnen fordern Gleichberechtigung “ präsentiert. Diese wurden im Rahmen der Veranstaltung „Frauen in Führungspositionen auf dem Weg nach oben: Wissenschaft und Unternehmen im Dialog “, die mit dem Deutschen Institut für Wirtschaftsforschung (DIW Berlin) und dem Wissenschaftszentrum Berlin für Sozialforschung (WZB) gemeinsam organisiert wurde, vorgestellt.

In den vergangenen vier Jahren hat der djb über 300 Hauptversammlungen besucht und vor mehr als 400.000 Aktionärinnen und Aktionären Fragen zu Frauen in Führungspositionen an Vorstände und Aufsichtsräte gestellt. Die Bilanz des mit Vorlage dieser Studie beendeten Projektes ist ernüchternd. Ramona Pisal, Präsidentin des djb: „Frauen stecken in den unteren Führungsebenen wie in einer Lehmschicht fest. Es fehlt ganz überwiegend an ernsthaften Bekenntnissen der Unternehmen zu mehr Frauen in Führungspositionen und in den Vorständen sowieso - vor allen Dingen fehlt es jedoch an zielführenden und nachhaltigen Aktivitäten, um diese Situation grundlegend zu ändern. Der djb hält deshalb eine gesetzliche Regelung, wie sie jüngst in den Koalitionsvereinbarungen für die Aufsichtsräte beschlossen wurde, auch für Führungspositionen und Vorstände für unerlässlich.“

Das Einführungskapitel zum Projekt „Aktionärinnen fordern Gleichberechtigung “ und das Kapitel „Fazit und Forderungen“, Kernstück der Studie, sind hier vollständig abgedruckt. Darüber hinaus ist die Rede von Ramona Pisal, die sie anlässlich der Vorstellung der Studie am 28. November 2013 im WZB gehalten hat, abgedruckt.
Auf der Veranstaltung haben sich die eingeladenen Personalvorstände, Prof. Dr. Marion Schick, Deutsche Telekom AG, und Wilfried Porth, Daimler AG, sowie die Personalverantwortlichen Peter Dollhausen, ThyssenKrupp AG und Dr. Christian Finckh, Allianz SE, zu mehr Frauen in Führungspositionen bekannt. Mit dem Slogan „Die Zukunft ist weiblicher“ sprachen sich die Vertreterinnen und Vertreter aus Wirtschaft, Wissenschaft und Zivilgesellschaft gemeinsam für gleichberechtigte Karrierechancen für Frauen und Männer in deutschen Unternehmen aus. Auszüge aus der vor Ort geführten Podiumsdiskussion über die Ergebnisse der Studie sowie daraus abgeleitete Handlungsempfehlungen und die prägnante Einführungsrede von PD Dr. Elke Holst, Forschungsdirektorin DIW Berlin, können ebenfalls hier nachgelesen werden.

Parallel zur Vorstellung der Abschlusspublikation im WZB präsentierte Manuela Schauerhammer, ehemalige Mitarbeiterin im Projekt „Aktionärinnen fordern Gleichberechtigung“, das Projekt und seine Ergebnisse auf eine ganz besondere Weise. Auf dem Diversity Slam, welcher im Rahmen der von Tagesspiegel und Charta der Vielfalt organisierten Diversity-Konferenz ebenfalls am 28. November 2013 erstmalig stattfand, konnte sich der djb als eines von vier Wettbewerbsbeiträgen qualifizieren. Ein kurzer Bericht über dieses außergewöhnliche Ereignis findet sich ebenfalls in dieser Ausgabe. 


\section{Das Projekt}

Was im Jahr 2009 mit dem Besuch von drei Hauptversammlungen beginnt, entwickelt sich durch großen Zuspruch und Solidarität innerhalb des Deutschen Juristinnenbundes e.V. (djb) schnell zu der "größten Aktion, die Deutschlands Hauptversammlungsbesucher je erlebt haben "1. 300 Hauptversammlungen in vier Jahren - jedes Jahr besucht der djb alle 30 DAX-Unternehmen sowie 45 weitere börsennotierte Unternehmen.

„Ein Blick ins Gesetz erleichtert die Rechtsfindung.“ - Diesem Credo folgend hatte Rechtsanwältin und Notarin Mechtild Düsing in Erfahrung gebracht, dass eine einzige Aktie einerseits erforderlich, andererseits aber auch ausreichend ist, um vom Auskunftsrecht nach $\$ 131$ Aktiengesetz (AktG) Gebrauch zu machen. Dieser schreibt vor, dass der Vorstand Aktionärinnen und Aktionären auf Verlangen in der Hauptversammlung Auskunft über Angelegenheiten der Gesellschaft geben muss. Auf dieser Idee basiert das Projekt „Aktionärinnen fordern Gleichberechtigung " des djb, das auf unkonventionelle und innovative Weise sowohl die Verantwortlichen in den Unternehmen als auch die Anteilseignerinnen und Anteilseigner anspricht.

Die Fragen des djb thematisieren den geringen Frauenanteil in Führungspositionen und alle damit zusammenhängenden Entscheidungen und Maßnahmen der Unternehmen. Dabei begleitet das Projekt die Unternehmen kritisch bei ihrem Umgang mit den Empfehlungen des Deutschen Corporate Governance Kodex (DCGK) und der Selbstverpflichtung der 30 DAX-Unternehmen vom 17. Oktober 2011. Das Projekt ist Teil des Stufenplanes „Mehr Frauen in Führungspositionen“ des Bundesministeriums für Familie, Senioren, Frauen und Jugend (BMFSFJ) und wird von diesem finanziell gefördert. Der djb stellt die „Womenpower" in Form von Aktionärinnen und Teilnehmerinnen und leistet die organisatorische Arbeit. An die 160 Teilnehmerinnen und 60 Aktionärinnen und Aktionäre, die ihre Aktien zur Verfügung stellen, unterstützen das Projekt.

\section{Im Laufe des Projekts \\ sprechen die Teilnehmerinnen vor mehr als 400.000 Aktionärinnen und Aktionären auf über 300 Hauptversammlungen.}

Ziel ist es, durch die Besuche von Hauptversammlungen, begleitet von umfangreicher Pressearbeit, Aufmerksamkeit für dieses brisante Thema zu erregen und durch wiederholte Teilnahme und Stellen der Fragen zu Frauen in Führungspositionen einen erheblichen Rechtfertigungsdruck seitens der Unternehmen aufzubauen. Beide Ziele werden vom djb erreicht.

Die Auftritte von Projektteilnehmerinnen rufen dabei oft kontroverse und auch emotionale Reaktionen im Publikum hervor. Der djb hat damit den Diskurs zum Thema „Frauen in Führungspositionen“ entscheidend beeinflusst und über Jahre mit geführt. Öffentlichkeitswirkung und Rechtfertigungsdruck seitens der Unternehmen wurden durch über vierzig Pressemitteilungen und mehr als 220 Medienberichte über das Projekt erzielt.
Ausgangssituation ist 2009 ein Frauenanteil in den 30 DAXVorständen von unter einem Prozent und in deren Aufsichtsräten auf Anteilseignerseite von 6,5 Prozent. ${ }^{2}$ Heute liegen diese Anteile bei über sieben bzw. über 18 Prozent. Der djb hat zu dieser Entwicklung durch das Projekt beigetragen.

Dem djb geht es aber nicht allein um die Erhöhung des Frauenanteils in den Vorständen und Aufsichtsräten.

Ziel des djb ist es,

den Anteil von Frauen in Führungspositionen auf allen Führungsebenen zu erhöhen.

Die genaue Anzahl von Frauen in Vorständen und Aufsichtsräten kann jeweils in den Geschäftsberichten der Unternehmen ermittelt werden. Das Alleinstellungsmerkmal des Projekts ist es hingegen, über das Auskunftsrecht Daten zu generieren, die selten veröffentlicht werden. Dazu zählt unter anderem der Anteil von Frauen auf den einzelnen Führungsebenen unterhalb des Vorstands in Deutschland. Darüber hinaus können auf den Hauptversammlungen Ziele, Strategien und Maßnahmen, aber auch Meinungen und Einstellungen der Unternehmensleitungen in Erfahrung gebracht und daraus Tendenzen für die Zukunft abgeleitet werden.

Die Befragungen der Unternehmen von 2010 bis 2012 sind bisher in drei Studien ${ }^{3}$ veröffentlicht. Neben der Auswertung selbst werden dort in Gastbeiträgen wichtige Aspekte des Themas vertieft. So werden in der Studie 2010 Diversity im DCGK, die Berichtspflichten im Lagebericht nach $\$ 289$ Abs. 3 HGB und die gesetzliche Quotierung von Aufsichtsräten dargestellt. 2011 gibt es Beiträge zur Zulässigkeit der Quotierung von Vorständen, zu Entwicklungen auf europäischer Ebene sowie Maßnahmen und Erfahrungen im Ausland. 2012 kommen externe Beiträge von der Bundesregierung, den Unternehmen selbst, Personalberatungen und Initiativen von Aktionärinnen und Aktionären sowie andere Initiativen, die sich zum Thema positionieren, hinzu. Auch wissenschaftliche Beiträge zur Rolle junger Frauen in MINT-Berufen und zur medialen Sichtbarkeit von Frauen werden in den Studien publiziert. Die hier vorliegende vierte Auswertung stellt eine Zusammenfassung unter Einbeziehung der aktuellen Daten für 2013 dar und zieht das Fazit.

\section{Bei fünf Veranstaltungen und in nunmehr vier Studien}

präsentiert das Projekt die Ergebnisse. Verteilt wurden bisher über 11.000 Exemplare.

1 Kessler, Gregor/Smolka, Klaus-Max: Hohe Nachfragequote, in: Financial Times Deutschland v. 10.5.2010.

2 Aktionärinnen fordern Gleichberechtigung, BMFSFJ Berlin, 2010, S. 49f.

3 Aktionärinnen fordern Gleichberechtigung, BMFSFJ Berlin, 2010, 2011, 2012. 
Angesichts der ernüchternden Ergebnisse der Befragungen kann nicht davon gesprochen werden, dass eine Gleichstellung von Frauen und Männern in der Wirtschaft bereits erreicht wäre oder ohne weiteres Zutun erreicht werden könnte. Das Projekt endet in diesem Jahr. Der djb plädiert daher für eine gesetzliche Regelung. Solange diese auf sich warten lässt, wäre es wünschenswert, den Besuch von Hauptversammlungen ehrenamtlich fortzuführen. Aktionärinnen und Bevollmächtigte, die hieran Interesse haben, sollten sich zeitnah vor der Hauptversammlung über das Unternehmen informieren und kritische Punkte auf der Hauptversammlung ansprechen möglichst jedes Jahr. Als Vorlage können die Fragen dienen, die im Rahmen des Projekts entwickelt wurden und öffentlich zugänglich sind.

Wer nichts fordert, bekommt auch nichts - so die Erfahrung. Der Druck auf die Unternehmen, sich gegenüber der Öffentlichkeit zu erklären, muss zwingend aufrechterhalten werden.

Das Ziel ist noch nicht erreicht, aber näher gerückt: die tatsächliche Gleichstellung von Frauen und Männern. Sie muss weiter mit Nachdruck eingefordert werden!

\section{Fazit und Forderungen}

Die Veränderungen in den Führungspositionen, Vorständen und Aufsichtsräten sind zu gering. Es mangelt an Strategien, die, getragen vom nachhaltigen Bekenntnis des höheren Managements zu mehr Frauen, signifikante Fortschritte erzielen. Der Deutsche Corporate Governance Kodex (DCGK) führt nicht zu Zielsetzungen der Unternehmen für Führungspositionen und den Vorstand. Die freiwilligen Selbstverpflichtungen der Unternehmen sind unzureichend, sie gelten bisher nur für die 30 DAX-Unternehmen und liegen oft unterhalb des Frauenanteils an der Belegschaft.

Es bestehen deshalb Zweifel an der Wirksamkeit dieser Instrumente.

\section{Der djb fordert von der Politik die}

Einführung einer Frauenquote von mindestens 40 Prozent für Führungspositionen, Vorstände und Aufsichtsräte.

Eine signifikante Erhöhung des Frauenanteils in den Führungspositionen, Vorständen und Aufsichtsräten kann nach Einschätzung des djb nur durch eine gesetzliche Regelung erfolgen.

Der DCGK kann für die Unternehmen zur Erreichung ihrer Ziele für mehr Frauen in den Führungspositionen, Vorständen und Aufsichtsräten ein nützliches Instrument sein.

\section{Der djb fordert jedoch von der Regierungskommission des DCGK die}

Konkretisierung der Empfehlungen zu den Führungspositionen und Vorständen.

Die Empfehlungen des DCGK zu den Führungspositionen (Ziff. 4.1.5) und zu den Vorständen (Ziff. 5.1.2) müssen analog zu den Vorgaben zum Aufsichtsrat (Ziff. 5.4.1) angepasst werden: „[...] Der Aufsichtsrat soll für seine Zusammensetzung konkrete Ziele benennen, [...]. Diese konkreten Ziele sollen insbesondere eine angemessene Beteiligung von Frauen vorsehen. [...] Die Zielsetzung des Aufsichtsrats und der Stand der Umsetzung sollen im Corporate Governance Bericht veröffentlicht werden."

Darüber hinaus sind die Empfehlungen zu den Führungspositionen, Vorständen und Aufsichtsräten dahingehend zu konkretisieren, dass die formulierten Ziele gleichzeitig mit einem festen Zeitplan verbunden sind.
Eine nachhaltige Wirkung des DCGK kann jedoch nur durch eine konsequente und übereinstimmende Befolgung der Empfehlungen durch die Unternehmen erreicht werden.

Der djb fordert deshalb von den Unternehmen die Einbindung der Ziele für mehr Frauen in den Führungspositionen, Vorständen und Aufsichtsräten in ihre Unternehmenspolitik und -kultur.

Die Unternehmen müssen die Erhöhung des Frauenanteils in Führungspositionen, Vorständen und Aufsichtsräten als festen Bestandteil ihrer strategischen Ausrichtung betrachten und konkrete Maßnahmen und Ziele in allen Bereichen und auf allen Ebenen des Unternehmens formulieren. Dazu gehört insbesondere die glaubhafte und engagierte Unterstützung durch den Vorstand, ohne die ein nachhaltiger Wandel der Unternehmenskultur nicht zu bewerkstelligen ist.

Der djb hat im Rahmen des Projekts „Aktionärinnen fordern Gleichberechtigung “ folgende Ergebnisse gewonnen und empfiehlt auf der Grundlage der bestehenden Regelungen:

\section{Führungspositionen}

\section{Wenig ambitionierte Zielsetzungen für mehr Frauen in Führungspositionen}

Der DCGK ist in Bezug auf Frauen in Führungspositionen nicht konkret genug und verfehlt daher seine Wirkung. Erst die Initiative der Bundesregierung vom 17. Oktober 2011 war Anlass für fast alle 30 DAX-Unternehmen, Selbstverpflichtungen hinsichtlich eines anvisierten Anteils von Frauen in Führungspositionen einzugehen. Acht der weiteren börsennotierten Unternehmen sind diesem Beispiel gefolgt. Die Ziele sind jedoch zu niedrig angesetzt: Zwei Drittel der Unternehmen bleiben mit den Zielsetzungen hinter dem Anteil von Frauen an den Beschäftigten zurück. Die wenig ehrgeizigen Zielsetzungen der Unternehmen schlagen sich auch im durchschnittlichen Anstieg des Anteils von Frauen an Führungspositionen in Deutschland nieder: Diesen Anteil hat der djb anhand der bisher veröffentlichten Angaben der 30 DAX-Unternehmen in den Statusberichten errechnet. Er liegt in den Vergleichsjahren von 2010 zu 2011 und 2011 zu 2012 bei jeweils unter einem Prozentpunkt. 


\section{Je höher die Führungsebene, desto niedriger der Frauenanteil}

Für die vom djb besuchten Unternehmen gilt weiterhin, dass die Frauenanteile abnehmen, je höher die Führungsebene im Unternehmen liegt. Deshalb fragt der djb 2013 erstmals nach den prozentualen Anteilen von Frauen und Männern an den Beförderungen in die erste und zweite Führungsebene. Nur wenige Unternehmen geben verwertbare Antworten. Der nach wie vor geringe Anteil von Frauen an den Beförderungen 2012 spricht dagegen, dass sie in absehbarer Zeit angemessen auf den oberen Führungsebenen vertreten sein werden und sich die Lücke zwischen dem Frauenanteil an den Beschäftigten und demjenigen in Führungspositionen schnell schließen wird. Die Führungsebenen sind für Frauen nicht durchlässig genug, um ihnen den Aufstieg in das oberste Management zu ermöglichen.

Der djb empfiehlt der Politik die

Ausdehnung der Selbstverpflichtungen auf weitere Aktienindizes Die Aufforderung der Bundesregierung, Selbstverpflichtungen. einzugehen, sollte auf die 50 MDAX-, 30 TecDAX-, 50 SDAXund weitere börsennotierte Unternehmen ausgedehnt werden.

- Der djb fordert von den Unternehmen

ehrgeizigere Selbstverpflichtungen für mehr Frauen in Führungspositionen.

Unternehmen müssen im Rahmen ihrer Selbstverpflichtungen Zielangaben deutlich höher ansetzen und sollten sich dafür mindestens an der Höhe des Frauenanteils an den Beschäftigten orientieren; sofern Letzteres sehr gering ist, sollten für Frauen in Führungspositionen dennoch darüber hinausgehende Ziele angestrebt werden, da zu berücksichtigen ist, dass der Anteil der Absolventinnen in wirtschafts- und rechtswissenschaftlichen Studiengängen dem der Männer entspricht. Insbesondere werden aus diesen Studiengängen viele Führungspositionen besetzt.

- Der djb empfiehlt den Unternehmen die

überproportionale Beförderung von Frauen in höhere Führungspositionen.

Um die Lücke zwischen dem Frauenanteil an den Beschäftigten und demjenigen in Führungspositionen zu schließen, sollten gleich qualifizierte Frauen in den nächsten Jahren überproportional stark bei Beförderungen in höhere Führungspositionen berücksichtigt werden.

\section{Mangelhafte Veröffentlichung frauenspezifischer Daten in den Lageberichten}

Obwohl Unternehmen Daten zum Frauenanteil an der Belegschaft und auf den einzelnen Führungsebenen in Deutschland erheben, fehlen diese Angaben nach wie vor in den Lageberichten. Über drei Viertel der 30 DAX-Unternehmen veröffentlichen gar keine frauenspezifischen Daten für Deutschland. Hier fehlt es eindeutig an Transparenz, da so nicht erkennbar wird, wie sich die Zahlen über die Jahre entwickeln und was sich auf den einzelnen Führungsebenen verändert.

\section{Divergenz zwischen abgefragten und in den Statusberichten der 30 DAX-Unternehmen angegebenen Daten}

Die Angaben, die die Unternehmen auf den Hauptversammlungen machen, weichen von den Angaben in den Statusberichten, welche die 30 DAX-Unternehmen seit dem Quotengipfel vom 17. Oktober 2011 jährlich veröffentlichen, ab. Beim Datenabgleich ergeben sich in den Statusberichten bei annähernd der Hälfte der 30 DAX-Unternehmen höhere Werte als bei denjenigen, die die Unternehmen auf den Hauptversammlungen angeben. Da die Führungsebenen in den Statusberichten nicht gesondert aufgeführt werden, lässt sich nicht feststellen, woraus sich diese - zum Teil erheblichen - Differenzen ergeben.

Der djb empfiehlt die

Veröffentlichung von frauenspezifischen Daten für die einzelnen Führungsebenen.

Die Unternehmen sollten die von ihnen bereits erhobenen Daten zu den Frauenanteilen an den Beschäftigten und auf den einzelnen Führungsebenen unterhalb des Vorstands für Deutschland im Lagebericht veröffentlichen, um Transparenz herzustellen.

Begleitend sollte der Gesetzgeber die in $\S 289$ Abs. 3 HGB verankerte Berichtspflicht der Unternehmen klarstellend folgendermaßen ergänzen, um auf eine Veröffentlichung der Daten hinzuwirken: „[...] Der Anteil der Frauen an der Gesamtzahl der Arbeitnehmerinnen und Arbeitnehmer und Organmitglieder auf den verschiedenen Hierarchieebenen, insbesondere am unteren, mittleren und Top-Management des Unternehmens, ist für die Lage der Gesellschaft immer von Bedeutung." Alternativ könnte vom Gesetzgeber ein neuer Absatz за folgenden Wortlauts eingefügt werden: „(3а) Absatz 1 Satz 3 gilt entsprechend für den Anteil der Frauen an der Gesamtzahl der Arbeitnehmerinnen und Arbeitnehmer und Organmitglieder auf den verschiedenen Hierarchieebenen, insbesondere am unteren, mittleren und Top-Management des Unternehmens."4

Die Politik sollte sich dafür einsetzen, dass die Frauenanteile auf den einzelnen Führungsebenen auch in den jährlichen Statusberichten der 30 DAX-Unternehmen ausgewiesen werden.

\section{Unzureichende Evaluation von Personalentwicklungspro- grammen}

Die Unternehmen haben bereits gute Strukturen für die Personalentwicklung geschaffen, sowohl was das Angebot an Programmen als auch die Evaluation, die Verantwortlichkeit und die Anbindung an den Vorstand und Aufsichtsrat betrifft. Diese Maßnahmen haben aber nicht zu einer signifikanten Erhöhung des Frauenanteils in Führungspositionen geführt. Ein möglicher Grund hierfür könnte sein, dass zu wenige Unternehmen erfassen, wie viele Frauen an Personalentwicklungsprogram-

4 Schmidt, Marlene: Novellierung des $\S 289$ HGB und flankierende Maßnahmen, in: Aktionärinnen fordern Gleichberechtigung, BMFSFJ Berlin, 2010, S. 106. 
men teilnehmen; noch viel seltener wird die Altersstruktur von Teilnehmerinnen an den Programmen erhoben. Darüber hinaus sind die Beförderungskriterien nicht mehr zeitgemäß, weil sie nicht auf die Lebensphasen von Frauen und Männern abgestimmt sind.

Der djb empfiehlt den Unternehmen die regelmäßige Evaluation der Personalentwicklungsprogramme. Personalentwicklungsprogramme sind hinsichtlich ihrer Qualität regelmäßig zu evaluieren. Hierfür sollten Ziele definiert und anhand von Indikatoren überprüft werden. Insbesondere ist zu berücksichtigen, wie viele Frauen an den Maßnahmen teilnehmen und wie viele davon unter bzw. über 40 Jahre alt sind. Mithilfe dieser Daten kann festgestellt werden, wann es zu Karrierebrüchen von Frauen kommt. Eine regelmäßige Berichterstattung der Verantwortlichen an den Vorstand muss gewährleistet sein. - Der djb empfiehlt den Unternehmen außerdem die Ermöglichung von lebensphasenorientierten Karrieren Beförderungskriterien sind auf ihre mittelbare Diskriminierung von Frauen zu überprüfen. Da Kriterien bisher in bestimmten Lebensphasen erfüllt werden müssen, ist dafür zu sorgen, dass Frauen diesen auch in anderen Lebensphasen entsprechen können. Tradierte Karrieremuster und vorhandene Personalentwicklungskonzepte in den Unternehmen sind unter der Berücksichtigung von lebensphasenorientierten Karriereverläufen von Frauen und Männern zu überprüfen und entsprechend neu zu gestalten

\section{Zu geringe Repräsentanz von Frauen in Besetzungsgremien}

Frauen waren 2012 noch zu selten in Besetzungsgremien vertreten, in denen Personalverantwortliche über die Besetzung von Führungspositionen entscheiden. Ihre Präsenz war zudem kaum durch interne Strukturen sichergestellt.

\section{Der djb empfiehlt den Unternehmen eine} verstärkte Präsenz von Frauen in Besetzungsgremien. Frauen müssen in Besetzungsgremien angemessen vertreten sein. Es ist sicherzustellen, dass jeweils mindestens eine personalverantwortliche Frau über die Besetzung von Führungspositionen mitentscheidet.

\section{Anreiz Bonussystem selten genutzt}

Zielvereinbarungen zur Erhöhung des Frauenanteils werden noch zu selten an die Vergütung der Vorstände und des höheren Managements gekoppelt.

Der djb empfiehlt den Unternehmen die Kopplung der Ziele an das unternehmensinterne Vergütungssystem.

Die Unternehmen sollten Zielvereinbarungen zur Erhöhung des Frauenanteils an die Vergütung der Vorstände und des höheren Managements koppeln. Dies trägt zu einer nachhaltigen Beförderungsstrategie von Frauen in Führungspositionen bei, da monetäre Anreize gesetzt werden.

\section{Keine Strategie bei der Kommunikation des Unternehmens- wandels}

Die Unternehmen verfügen über eine Vielzahl von Kommunikationsmitteln und -weisen zum Thema „Frauen in Führungspositionen “ und dem damit angestrebten Wandel der Unternehmenskultur. Dennoch lässt sich häufig keine zielführende Strategie zur Erhöhung des Anteils von Frauen in Führungspositionen ableiten. Nur die wenigsten Unternehmen bestimmen Kennzahlen, anhand derer der Fortschritt und Erfüllungsgrad ihrer Zielsetzung auch gemessen werden kann. Der in der Mehrzahl befürwortete Wandel kann so nicht herbeigeführt werden.

Der djb empfiehlt den Unternehmen die strategische Ausrichtung der Unternehmenskommunikation an allen Mitarbeitenden.

Das Thema „Frauen in Führungspositionen“ sollte von den Unternehmen in gleichem Maße gegenüber Frauen und Männern kommuniziert werden, um eine einseitige Kategorisierung als Frauenthema zu vermeiden. Bestehende Maßnahmen der Unternehmen sollten hinsichtlich ihres Adressatenkreises überprüft werden. Den Kommunikationsmaßnahmen muss eine zielführende Strategie hinsichtlich eines Unternehmenswandels zu mehr Frauen in Führungspositionen zugrunde gelegt werden. Der so angestrebte Wandel muss anhand definierter Kennzahlen überprüfbar sein.

\section{Kein betriebswirtschaftliches Kalkül für mehr Frauen in Füh- rungspositionen}

Der wirtschaftliche Vorteil von Geschlechtervielfalt wird nur von wenigen Unternehmen (an-)erkannt. In der Folge mangelt es an einer fundierten strategischen Ausrichtung der Unternehmen, die vor allem auch den Entwurf eines betriebswirtschaftlichen Geschäftsszenarios mit einschließt. Noch zu selten wird kalkuliert, ob und inwiefern ein höherer Anteil von Frauen in Führungspositionen zu einem Mehrwert für das Unternehmen und einem Wettbewerbsvorteil gegenüber anderen Unternehmen, auf dem Markt und bei Kundinnen und Kunden beiträgt.

Der djb empfiehlt den Unternehmen die
Behandlung der Thematik „Frauen in Führungspositionen“
als Business Case.
Die Erhöhung des Anteils von Frauen in Führungspositionen
muss zur „Chefsache“ werden. Nur ein starkes und nachhalti-
ges Bekenntnis der Vorstände führt zu einem höheren Stellen-
wert des Themas im Unternehmen und zu dessen Akzeptanz
durch die Mitarbeitenden. Darüber hinaus sollte die Zielset-
zung des Unternehmens „Mehr Frauen in Führungspositio-
nen“- wie alle anderen Themen - auch als betriebswirtschaft-
liches Geschäftsszenario behandelt werden. Das Ziel sollte mit
der bestehenden Unternehmensstrategie und -kultur ver-
knüpft werden, um mögliche Wettbewerbsvorteile für das Un-
ternehmen zu eruieren.




\section{Vorstände}

\section{Keine Ziele für Frauen in Vorständen}

Frauen fehlen auf der Agenda der Unternehmen, wenn es um die Besetzung von Vorstandspositionen geht. Von zwei Ausnahmen unter den vom djb besuchten Unternehmen abgesehen definieren die Unternehmen 2013 weder in ihrem Corporate Governance Bericht noch auf Nachfrage Ziele für den Frauenanteil im Vorstand. Der DCGK entfaltet in Bezug auf die Vorstände für Frauen also keine Wirkung. Er ist nicht konkret genug und wird von den Unternehmen nicht so verstanden, dass auch für den Vorstand messbare Ziele zu formulieren seien. Der Vergleich zu den Aufsichtsräten zeigt: Empfiehlt der DCGK Ziele, werden diese von den Unternehmen - wenn auch mit sehr unterschiedlichen Vorgaben - aufgenommen; bleibt der Kodex hingegen allgemein, werden keine konkreten Ziele formuliert.

\section{Unzureichende Maßnahmen für mehr Frauen in den Vorständen} Grundsätzlich rekrutieren Unternehmen ihre Vorstände bevorzugt und überwiegend intern. Tatsächlich wurden aber mehr als die Hälfte der seit 2010 in den Vorstand eines DAX-Unternehmens berufenen Frauen extern rekrutiert. Daraus kann geschlossen werden, dass es versäumt wurde, weibliche Talente in den Unternehmen selbst zu identifizieren und zu fördern.

Die Maßnahmen der Unternehmen, Frauen für den Vorstand zu entwickeln, sind nicht ausreichend. Es fehlt weiterhin an Anforderungsprofilen, öffentlichen Stellenausschreibungen und einer systematischen Suche nach Frauen für Vorstandspositionen: Nur circa ein Viertel aller befragten Unternehmen hat 2013 bei Neubesetzungen gezielt nach Frauen gesucht.

\section{Keine nachhaltige Entwicklung bei der Besetzung von Vor- standspositionen mit Frauen}

Der Aufwärtstrend bei der Besetzung von Vorstandspositionen mit Frauen aus dem vorhergehenden Jahr setzt sich 2013 nicht fort. Dies wird besonders deutlich, wenn die bisherigen Neubestellungen bzw. Verlängerungen bestehender Vorstandsverträge berücksichtigt werden. Nur jede 14 . Neu- bzw. Wiederbestellung in den Vorständen der 30 DAX- bzw. der 46 weiteren Unternehmen betrifft eine Frau. Damit sinkt der weibliche Anteil an Vorstandspositionen bei den 30 DAX-Unternehmen auf 7,4 Prozent. Bei den weiteren börsennotierten Unternehmen konnte 2013 der Frauenanteil in den Vorständen minimal auf 4,6 Prozent gesteigert werden. Die Entwicklungen zeigen, dass Besetzungen in den Vorständen mit Frauen auf keiner nachhaltigen Strategie des Managements beruhen. Der Aufwärtstrend aus dem vorhergehenden Jahr scheint mit der politischen Diskussion um die Einführung einer gesetzlichen Frauenquote zusammenzuhängen. Fällt der politische Druck weg, stagniert auch hier die Entwicklung.

Der djb fordert von den Unternehmen eine Agenda für mehr Frauen in den Vorständen. Die Unternehmen müssen verstärkt die interne Entwicklung von Frauen für Vorstandspositionen fördern. Darüber hinaus sollten sie öffentlich zugängliche Anforderungsprofile erstellen, Vakanzen öffentlich ausschreiben und bei Neubesetzungen gezielt nach Frauen suchen.

\section{Aufsichtsräte}

Anspruchslose Zielsetzungen für mehr Frauen in Aufsichtsräten Angestoßen durch den DCGK haben sich viele Unternehmen Ziele für eine aus ihrer Sicht angemessene Beteiligung von Frauen in den Aufsichtsräten gesetzt. 2013 erklären allerdings noch drei der 30 DAX-Unternehmen und 18 der weiteren börsennotierten Unternehmen, dass sie in Bezug auf Diversity vom DCGK abweichen. Insgesamt fünf der 30 DAX- und 27 der börsennotierten Unternehmen nennen 2013 keine Ziele.

Die Ziele der Unternehmen sind auch weiterhin zu wenig ambitioniert. Nur ein Unternehmen hat sich als Ziel eine Frauenquote von 40 Prozent im Aufsichtsrat gesetzt. Jeweils eines der 30 DAX- und eines der weiteren börsennotierten Unternehmen haben bereits die 40-Prozent-Marke überschritten. Einen Frauenanteil um 30 Prozent haben aktuell circa ein Drittel der 30 DAX-Unternehmen bereits erreicht oder als Zielmarke formuliert; bei den weiteren börsennotierten Unternehmen sind es nur acht Unternehmen.

Entsprechenserklärungen erlauben ohne nähere Recherchen in den Corporate Governance Berichten und ohne Kenntnis der Unternehmensdaten keine Aussage darüber, ob Ziele für eine angemessene Beteiligung von Frauen in den Aufsichtsräten in Form von Zahlen oder Prozenten formuliert werden und ob die Ziele angemessen sind oder bereits erreicht wurden.

Circa zwei Drittel der 30 DAX-Unternehmen und circa ein Viertel der weiteren börsennotierten Unternehmen haben ihr selbst gesetztes Ziel bereits erreicht. Mit einer zügigen weiteren Entwicklung kann aufgrund der Erklärungen der Unternehmen in ihren Corporate Governance Berichten und auf den Hauptversammlungen daher nicht gerechnet werden. Der Prozess stagniert sowohl hinsichtlich der Anzahl der Unternehmen, die sich Ziele setzen, als auch hinsichtlich der Größenordnung dieser Ziele.

\section{Der djb empfiehlt den Unternehmen} ambitionierte Zielsetzungen für Frauen in den Aufsichtsräten. Der DCGK ist in seinen Vorgaben zum Aufsichtsrat bereits konkretisiert worden. Dennoch verhindert die unterschiedliche Auslegung und Beurteilung der Unternehmen, was eine angemessene Berücksichtigung von Frauen ist, seine nachhaltige Wirkung. Die Unternehmen müssen ihre Zielsetzungen hinsichtlich ihrer Angemessenheit regelmäßig überprüfen und sich einen konkreten Zeitrahmen setzen.

\section{Keine Transparenz in den Auswahlverfahren}

Die Wahlvorschläge für die Aufsichtsräte sind nicht transparent. Es gibt nur selten auf das Unternehmen zugeschnittene Anforderungsprofile; die meisten Unternehmen zitieren 
lediglich die Anforderungen aus dem DCGK. Öffentliche Ausschreibungen fehlen. Während 2012 circa ein Drittel der untersuchten Unternehmen Personalberatungen in Anspruch nahm, werden diese 2013 nur noch von jedem zehnten Unternehmen genannt. Anfragen bei der Wirtschaftsprüferkammer oder in Datenbanken gibt es nicht. Dies zeigt, dass sich die Suchstrategien der Unternehmen nicht maßgeblich geändert haben und diese weiterhin überwiegend in internen Netzwerken suchen.

Der djb empfiehlt den Unternehmen

transparente Auswahlverfahren und eine Erweiterung des Bewerberkreises.

Für alle Aufsichtsratspositionen sind öffentlich zugängliche Anforderungsprofile zu erstellen und Vakanzen öffentlich auszuschreiben, um auch externe Bewerbungen zu ermöglichen. Die Unternehmen sollten verstärkt auch extern nach Aufsichtsratsmitgliedern suchen und sich nicht auf eigene Netzwerke beschränken.

\section{Keine Trendwende im „Superwahljahr“ 2013}

Zwar hat der Frauenanteil bei den Aufsichtsräten der 30 DAXUnternehmen die 20-Prozent-Marke 2013 erstmals überschritten, von einer Trendwende kann aber nicht die Rede sein. Weiterhin wird nur ein Drittel der Frauen von Anteilseignerseite gestellt. In diesem Jahr fanden in 20 der 30 DAX-Unternehmen Aufsichtsratswahlen auf Anteilseignerseite statt. Nur etwas mehr als ein Viertel der Positionen wurde mit Frauen besetzt. Im Vergleich zu den Vorjahren bleibt dieser Wert damit annähernd konstant.

Bei den 46 weiteren börsennotierten Unternehmen liegt der Frauenanteil in den Aufsichtsräten bei knapp über 16 Prozent. Die Verteilung der Frauen auf der Anteilseigner- und Arbeitnehmerseite ist annähernd ausgeglichen. In 26 der 46 Unternehmen fanden auch hier Aufsichtsratswahlen auf Anteilseignerseite statt. Nur knapp 15 Prozent der neugewählten Aufsichtsratsmitglieder sind weiblich. Das „Superwahljahr“ 2013 wurde nicht konsequent genutzt, um mehr Frauen in die Aufsichtsräte zu bringen.

\section{Unterrepräsentanz von Frauen nicht durch Berufsabschlüsse bedingt}

2010 und 2013 untersucht der djb die berufliche Qualifikation der Aufsichtsratsmitglieder auf Anteilseignerseite in den 30 DAX-Unternehmen. Die Überprüfung zeigt, dass circa 60 Prozent der Positionen mit Absolventinnen und Absolventen wirtschaftswissenschaftlicher und/oder juristischer Studienrichtungen besetzt sind. Technische und/oder naturwissenschaftliche Qualifikationen sind mit circa 20 Prozent deutlich weniger vertreten. Für die Vorstandspositionen der 30 DAX-Unternehmen stellt sich die Situation annähernd gleich dar. Dieses Ergebnis entkräftet die Aussage vieler Unternehmen, dass der Frauenanteil an Führungspositionen, insbesondere auch bei Vorstandsund Aufsichtsratspositionen, so gering sei, weil technische und naturwissenschaftliche Studiengänge Voraussetzung für diese Positionen seien und es schlichtweg an Frauen mit solchen Berufsabschlüssen fehle.

\section{Zu geringe Repräsentanz von Frauen in Aufsichtsratsausschüssen}

Die Präsenz von Frauen in Personal- und Nominierungsausschüssen ist weiterhin noch zu gering, als dass diese tatsächlich Einfluss auf die Besetzung von Vorstands- und Aufsichtsratspositionen ausüben können: Insgesamt beläuft sich ihr Anteil in den Personalausschüssen auf 14 Prozent bei den 30 DAX- und auf 12,7 Prozent bei den weiteren börsennotierten Unternehmen. In den Nominierungsausschüssen liegt der Frauenanteil bei 14,7 Prozent in den 30 DAX- bzw. bei 9,7 Prozent in den weiteren börsennotierten Unternehmen. Auch in den weiteren Aufsichtsratsauschüssen der 30 DAXUnternehmen erreicht der Frauenanteil nicht die 20-ProzentMarke.

Der djb empfiehlt den Unternehmen eine angemessene Vertretung von Frauen in den Aufsichtsratsausschüssen.

Unternehmen sollten analog zu den Vorgaben des DCGK bei der Zusammensetzung der Aufsichtsräte darauf achten, dass Frauen in allen Ausschüssen angemessen vertreten sind. In den Nominierungs- und Personalausschüssen muss jeweils mindestens eine Frau präsent sein. 